\title{
基于高分辨率与高光谱遥感影像的北亚热带马尾 松及次生落叶树种的分类
}

\author{
申 金金 曹 林 徐 婷 余光辉
}

南京林业大学南方现代林业协同创新中心, 南京 210037

摘 要 利用遥感数据开展森林资源树种的分类对森林资源的监测、森林可持续经营及生物多样性研究都有重要意义。该文 以江苏南部丘陵地区的北亚热带天然次生林为研究对象, 利用LiCHy (LiDAR、CCD、Hyperspectral)集成传感器同期获取的 高分辨率和高光谱数据, 进行冠幅识别和多个层次的树种分类: 首先, 对高分辨率影像进行基于边缘检测的多尺度分割, 提 取出单木冠幅; 其次, 对高光谱影像进行特征变量提取, 并对提取出的特征变量利用信息熵原理选取优化特征变量; 然后, 分别利用全部特征变量和经优化的重要特征变量对森林树种及森林类型进行预分类; 最后, 在预分类结果中加入单木冠幅信 息对森林树种及森林类型进行重分类, 并分析分类结果的精度。研究表明: 1)利用全部特征变量进行4个典型树种分类时, 总 体精度为 $64.6 \%$, Kappa系数为 0.493 ; 而针对森林类型的分类精度为 $81.1 \%$, Kappa系数为 0.584 。2)利用选取的优化特征变量分 类精度略低于利用全部特征变量的分类精度, 其中对 4 个典型树种分类时, 总体精度为 $62.9 \%$, Kappa系数为 0.459 ; 而针对森 林类型的分类精度为 $77.7 \%$, Kappa系数为 0.525 。通过集成传感器同期获取的高分辨率和高光谱数据可以有效地进行北亚热带 森林的树种分类及森林类型的划分。

关键词 北亚热带森林, 树种分类, 高分辨率数据, 高光谱数据, 单木冠幅提取

引用格式: 申金曹林, 徐婷, 余光辉 (2015). 基于高分辨率与高光谱遥感影像的北亚热带马尾松及次生落叶树种的分类. 植物生态学报, 39, 1125-1135. doi: 10.17521/cjpe.2015.0109

\section{Classification of Pinus massoniana and secondary deciduous tree species in northern sub- tropical region based on high resolution and hyperspectral remotely sensed data}

SHEN Xin, CAO Lin, XU Ting, and SHE Guang-Hui*

Co-innovation Center for Sustainable Forestry in Southern China, Nanjing Forestry University, Nanjing 210037, China

\begin{abstract}
Aims Using remote sensing data for tree species classification plays a key role in forestry resource monitoring, sustainable forest management and biodiversity research.

Methods This study used integrated sensor LiCHy (LiDAR, CCD and Hyperspectral) to obtain both the high resolution imagery and the hyperspectral data at the same time for the natural secondary forest in south Jiangsu hilly region. The data were used to identify the crown and to classify tree species at multiple levels. Firstly, tree crowns were selected by segmenting high-resolution imagery at multiple scales based on edge detection; secondly, characteristic variables of hyperspectral images were extracted, then optimization variables were selected based on the theory of information entropy. Tree species and forest types were classified using either all characteristic variables or optimization variables only. Finally, tree species and forest types were reclassified along with the tree crowns information, and the accuracy of classification was discussed.

Important findings Based on all available characteristic variables, the overall accuracy for four typical tree species classification was $64.6 \%$, and the Kappa coefficient was 0.493 . The overall accuracy for forest types classification was $81.1 \%$, and the Kappa coefficient was 0.584 . Based on optimization variables only, the overall accuracy for four typical tree species classification dropped to 62.9\%, and the Kappa coefficient was 0.459. The overall accuracy for forest types classification was 77.7\%, and the Kappa coefficient was 0.525. Obtaining both high resolution image and hyperspectral data at the same time by integrated sensor can increase overall accuracy in classifying forest types and tree species in northern subtropical forest.
\end{abstract}

收稿日期Received: 2015-03-17 接受日期Accepted: 2015-09-20

* 通讯作者Author for correspondence (E-mail: ghshe@njfu.com.cn) 
Key words northern subtropical forest, tree species classification, high resolution image, hyperspectral data, tree crowns

Citation: Shen X, Cao L, Xu T, She GH (2015). Classification of Pinus massoniana and secondary deciduous tree species in northern subtropical region based on high resolution and hyperspectral remotely sensed data. Chinese Journal of Plant Ecology, 39, 1125-1135. doi: 10.17521/cjpe.2015.0109

精确获取森林树种信息及其空间分布信息对于 理解森林生态系统的结构、功能及其演替, 以及生 物多样性的研究具有重要意义(张鼎华和林卿, 2000; 郭中伟等, 2001; 李宇吴, 2007)。同时, 树种 空间分布信息可用于森林生长模型和生态过程模型 的参数化, 指导和优化森林生态系统模拟(何美成, 2006)。常规的树种调查方法主要依赖于地面野外调 查及利用大比例尺航片人工判读等, 工作量通常较 大, 且不利于森林树种信息的更新。而遥感技术具 有宏观、动态和快速的特点, 可以弥补常规地面调 查方法的不足。

近年来, 高分辨率和高光谱遥感技术的发展, 为中到大尺度上的森林信息提取提供了更高空间和 光谱分辨率的数据源, 同时在冠幅提取和树种分类 中也具有很大潜力(王吉斌等, 2013)。然而, 以往的 研究往往基于单一数据源(如TM, ALOS, CASI等), 如王婧等(2013)利用ALOS多光谱影像对福建省三 明市将乐林场的树种进行分类; 且大多只针对主要 森林类型, 如李小梅等(2010)通过CHRIS/PROBA高 光谱影像, 对吉林省长白山地区进行纯林和混交林 的分类; 或只针对优势树种组, 如温一博和范文义 (2013)利用多时相的 TM数据对黑龙江省塔河县的 北寒带森林进行分类, 区分出当地森林中的 4 个优 势树种组。同时, 由于传统传感器数据获取能力的 限制, 基于同期获取的高分辨率与高光谱遥感影像 共同进行树种分类的研究也很少。

针对以上研究空白, 本研究探索基于同期获取 的高分辨率与高光谱遥感影像对北亚热带的典型树 种进行冠幅提取和多个层次的树种分类, 旨在通过 集成两个数据源的优势细化树种分类层次, 并进一 步提升分类精度。以江苏南部丘陵地区的北亚热带 天然次生林为研究对象, 利用 LiCHy (LiDAR, CCD 和Hyperspectral)集成传感器同期获取的高分辨率和 高光谱数据, 首先基于高分辨率数据和面向对象分 割方法进行冠幅识别, 然后基于高光谱数据提取的 空间细节和光谱特征并结合BP神经网络分类器进 行树种分类, 最后通过混淆矩阵验证精度, 从而为
基于遥感多源同期数据集成方法的冠幅自动提取和 树种识别探索有效的技术途径。

\section{1 材料和方法}

本研究技术路线如图1所示, 具体步骤在下文 中阐述。

\section{1 研究区概况}

研究区位于江苏省常熟市国营虞山林场 $\left(120.70^{\circ} \mathrm{E}, 31.67^{\circ} \mathrm{N}\right)$, 面积约 $1422 \mathrm{hm}^{2}$, 高程变化 范围为2-261 m; 研究区所处区域为亚热带季风气 候, 年降水量 $1062.5 \mathrm{~mm}$; 其森林类型属于亚热带 次生混交林, 可细分为针叶林、阔叶林和混交林。 其中主要针叶和阔叶落叶树种包含马尾松(Pinus massoniana)、麻栋(Quercus acutissima)、枫香树 (Liquidambar formosana) 和板栗 (Castanea mollissima) 等, 同时伴生部分常绿阔叶树种。

\section{2 遥感数据获取及预处理}

本研究使用的遥感数据源于LiCHy (LiDAR、 CCD、Hyperspectral)传感器系统(Pang et al., 2013), 数据获取时间为2013年8月17日。其中的高光谱数据 为AISA Eagle子传感器获取, 空间分辨率为 $0.6 \mathrm{~m}$, 波长范围为398.5-994.4 nm, 光谱范围覆盖64个波 段, 辐射分辨率为 12 bit。首先借助AISA Eagle传感 器的辐射定标参数对原始影像进行辐射定标, 将原 始影像的灰度值 $D N$ 转化为像元辐射亮度值, 然后 利用FLAASH大气校正模型和平场域校正模型将像 元辐射亮度值转化为地表反射率值。

高分辨率影像为LiCHy系统中的DigiCAM-60 子传感器在同一时间获取, 空间分辨率为 $0.1 \mathrm{~m}$, 辐 射分辨率为 8 bit。首先, 对影像进行几何精校正, 使 其配准到高光谱影像。选取320个同名像点, 采用二 次多项式进行校正, 并使得校正误差控制在1个像 元之内, 然后使用最邻近像元法进行重采样。

\section{3 地面调查数据}

根据2012年虞山林场森林资源调查数据中的树 种组成、年龄和立地分层选取 7 个 $30 \mathrm{~m} \times 30 \mathrm{~m}$ 的正 方形样地, 样地包括针叶林、阔叶林和混交林 


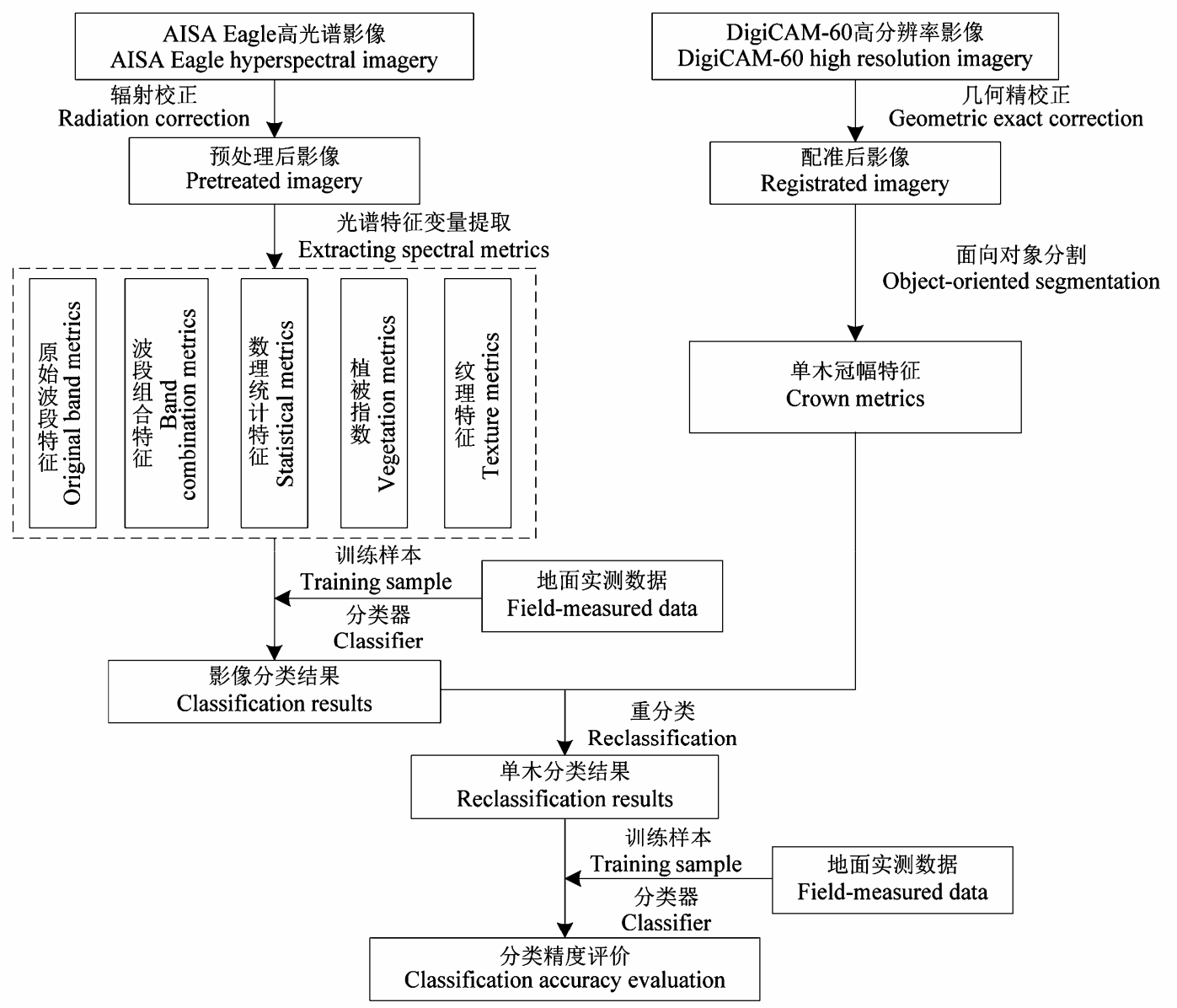

图1 集成高分辨率和高光谱遥感数据的树种分类技术路线。

Fig. 1 The technical route of tree species classification using high resolution and hyperspectral data.

表1 主要树种信息汇总表

Table 1 Summary of forest metrics for the four main tree species

\begin{tabular}{|c|c|c|c|c|c|c|c|c|c|c|c|c|}
\hline \multirow{2}{*}{$\begin{array}{l}\text { 林木参数 } \\
\text { Forest metrics }\end{array}$} & \multicolumn{3}{|c|}{ 马尾松 Pinus massoniana } & \multicolumn{3}{|c|}{ 麻栎 Quercus acutissima } & \multicolumn{3}{|c|}{ 板栗 Castanea mollissima } & \multicolumn{3}{|c|}{ 枫香树 Liquidambar formosana } \\
\hline & $\begin{array}{l}\text { 范围 } \\
\text { Range }\end{array}$ & $\begin{array}{l}\text { 平均值 } \\
\text { Mean }\end{array}$ & $\begin{array}{c}\text { 标准偏差 } \\
S D\end{array}$ & $\begin{array}{l}\text { 范围 } \\
\text { Range }\end{array}$ & $\begin{array}{l}\text { 平均值 } \\
\text { Mean }\end{array}$ & $\begin{array}{c}\text { 标准偏差 } \\
S D\end{array}$ & $\begin{array}{l}\text { 范围 } \\
\text { Range }\end{array}$ & $\begin{array}{l}\text { 平均值 } \\
\text { Mean }\end{array}$ & $\begin{array}{c}\text { 标准偏差 } \\
S D\end{array}$ & $\begin{array}{l}\text { 范围 } \\
\text { Range }\end{array}$ & $\begin{array}{l}\text { 平均值 } \\
\text { Mean }\end{array}$ & $\begin{array}{c}\text { 标准偏差 } \\
S D\end{array}$ \\
\hline 胸径 $D B H(\mathrm{~cm})$ & $8.6-26.7$ & 15.5 & 3.6 & $5.7-35.4$ & 16.1 & 7.0 & $13.0-39.2$ & 28.3 & 6.9 & $6.6-32.0$ & 15.6 & 6.6 \\
\hline 树高 Tree height (m) & $6.1-18.2$ & 10.1 & 1.8 & $6.0-18.7$ & 11.4 & 2.6 & $8.1-16.1$ & 12.5 & 1.9 & $5.9-19.5$ & 11.3 & 3.3 \\
\hline 冠幅半径 Crown radius (m) & $0.4-3.7$ & 1.3 & 0.5 & $0.7-6.3$ & 2.2 & 1.1 & $1.8-4.9$ & 3.0 & 0.7 & $0.5-4.1$ & 2.2 & 0.9 \\
\hline
\end{tabular}

$\overline{D B H}$, diameter at breast height; $S D$, standard deviation.

3种森林类型。样地调查时间为2013年8月, 样地的4 个角点、中心位置及子 $(1 / 4)$ 样地中心位置通过 Trimble GeoXH 6000 Handhelds手持GPS定位(结合 JSCROS广域差分系统获得亚米级定位精度)(Cao et al., 2014)。样地的方向和倾角通过罗盘仪测定, 边 界长度通过皮尺测量。在各个样地中, 记录胸径 $\geqslant 5$ $\mathrm{cm}$ 的每棵单木的树种、空间位置(即 $X$ 和 $Y$ 坐标: 通过 计算其相对子样地中心的距离和水平方向夹角), 并测量胸径、树高、冠幅、树冠垂直等级(CC, crown class)等因子。胸径采用围尺测量, 树高通过Vertex IV 超声波测高器获取, 冠幅则在 2 个主方向上使用 皮尺测量其投影距离(冠幅半径=两个主方向上的投 影距离之和除以 4$)$ 。 3 种森林类型内的 4 个主要树种 参数汇总于表 1 。

\section{4 单木冠幅特征提取及精度验证}

首先利用基于边缘检测的多尺度分割方法对校 正后的高分辨率影像进行面向对象分割, 并剔除背 景信息, 从而提取单木冠幅, 然后利用地面实测数 
据评价提取精度。提取精度包括探测位置准确性和 冠幅半径准确性。探测位置准确性采用分割树冠和 地面实测树冠中心点的距离算法做匹配, 即设置距 离阈值, 当地面实测冠幅中心点在分割冠幅半径 (阈值)之内时, 认为分割冠幅探测正确。另外, 考虑 到地面实测冠幅中心点是在地面测量, 与分割的冠 幅不在同一高度, 因此加入树干倾斜改正，将分割 冠幅半径加上 $2 \mathrm{~m}$ 作为探测距离的阈值。距离小于 探测阈值的探测位置准确。探测位置的准确性可以 用以下3个指标衡量:

$$
r=\frac{N t}{N t+N o}, \quad p=\frac{N t}{N t+N c}, \quad F=\frac{2(r \times p)}{r+p}
$$

式中, $r$ 为冠幅的探测率, $p$ 为探测出的冠幅的准确 率, $F$ 为总体精度(包含未探测出的树冠和探测出但 地面实测并不存在的树冠), $N t$ 为影像上探测出且与 地面实测相匹配的冠幅数量, No 为影像上未探测出 但地面实测存在的冠幅数量, $N_{C}$ 为影像上探测出但 地面实测并不存在的冠幅数量。对于冠幅半径的准 确性, 考虑冠幅的形态学特征, 将提取的单木冠幅 边界近似为圆形, 按照圆形面积计算公式反推出冠 幅半径。将地面实测冠幅半径与影像提取的冠幅半 径做回归分析计算冠幅半径提取的精度。

\section{5 光谱特征变量提取及优化}

在高光谱影像上对 4 个主要树种分别选取 50 个 冠层光谱反射率曲线, 通过平均光谱反射率曲线求 得 4 个树种冠层上表面平均反射率曲线, 见图2。可 见, 在近红外波段 4 个树种反射率差异很大。因此, 选择近红外波段的各波段作为原始波段特征变量。 另外, 通过对高光谱影像进行波段组合、多种植被 指数变换、主成分分析、独立成分分析、最小噪声 分离以及纹理分析, 共提取出 5 组共 47 个特征变量: 包括12个原始单波段特征、8个波段组合特征、9个 植被指数特征、 9 个数理统计特征以及 9 个纹理特征。 47 个特征变量的含义及计算公式见附录 1 。

根据Shannon信息论原理, 一幅 $k$ bit $(k$ 为辐射 分辨率)表示的图像 $x$ 的熵 $H(x)$ 为:

$$
H(x)=-\sum_{i=0}^{2^{k}-1} P_{i} \log _{2} P_{i}
$$

式中 $P_{i}$ 为图像像素灰度值为 $i$ 的概率。同理, $n$ 幅影 像的联合熵 $H\left(x_{1}, x_{2}, \ldots, x_{n}\right)$ 为:

$$
H\left(x_{1}, x_{2}, \cdots, x_{n}\right)=-\sum_{i_{1}, i_{2}, \cdots, i_{n}=0}^{2^{k}-1} P_{i_{1}, i_{2}, \cdots, i_{n}} \log _{2} P_{i_{1}, i_{2}, \cdots, i_{n}}
$$

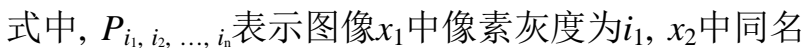
像素灰度为 $i_{2}, x_{3}$ 中同名像素灰度为 $i_{3}$, 以此类推, 一 直到 $x_{n}$ 中像素灰度为 $i_{n}$ 时的联合概率。通常, $H(x)$ 、 $H\left(x_{1}, x_{2}, \ldots x_{n}\right)$ 越大, 图像或图像组所含的信息越丰 富。根据信息熵原理, 在 47 个特征变量中选取了信 息熵最大的前 12 个特征变量作为优化特征变量。

\section{6 影像分类}

本研究采用 $\mathrm{BP}$ 神经网络对影像进行分类, $\mathrm{BP}$ 神 经网络是由大量处理单元相互连接并形成网络结构 的一种反向传播学习算法。网络开始训练时选用较 小的随机互联权值与内部阈值, 经过反复加载训练 样本并调整权值, 直到代价函数下降到可以接受的 容限值为止(骆剑承等, 2001)。BP神经网络算法是一 种将非线性多层感知系统的判决能力与均方误差函 数极小化的LMS算法相结合的产物。对于一个输入 样本 $p$, 其平方误差 $E_{p}$ 可定义为:

$$
E_{p}=\frac{1}{2} \sum_{k}\left(t_{k}-o_{k}\right)^{2}
$$

式中, $t_{k}$ 为输出层 $k$ 的期望输出, $o_{k}$ 为输出层 $k$ 的实际 输出。对于整个网络系统, 其总均方误差 $(E)$ 为:

$$
E=\frac{1}{q} \sum E_{q}
$$

其中 $q$ 为训练样本总数。网络训练的目的是找到一组 权值, 使E极小化。这里LMS算法用梯度下降法, 即 权重的增量正比于误差的负导数, 使得过程收玫。

本研究的分类共有 4 组, 第 1 组为使用全部特征 变量, 分 4 个树种进行分类; 第 2 组为使用优化特征 变量, 对 4 个树种进行再次分类; 第 3 组为使用全部 特征变量, 按森林类型进行分类; 第4组为使用优 化特征变量, 按森林类型再次进行分类。首先利用 全部特征变量(5组47个)进行分类, 对 4 个树种分别 选择训练区域, 进行训练学习, 再利用训练的模型 对未知区域进行分类。分类完成后加入单木冠幅信 息采用面积权限法进行重分类, 即冠幅面积区域内 占大多数面积的树种类型为整个冠幅的树种类型。 重分类后利用地面实测数据进行分类精度评价。然 后, 利用优化特征变量 (12个)对 4 个树种类型再次进 行分类, 分类方法为BP神经网络法, 分类完成后加 入单木冠幅信息进行重分类, 并进行分类精度评 价。其次, 利用全部特征变量(5组47个)对森林类型 进行分类, 分为针叶和阔叶两种森林类型, 分类方 法选用 $\mathrm{BP}$ 神经网络法, 分类后加入单木冠幅信息进 


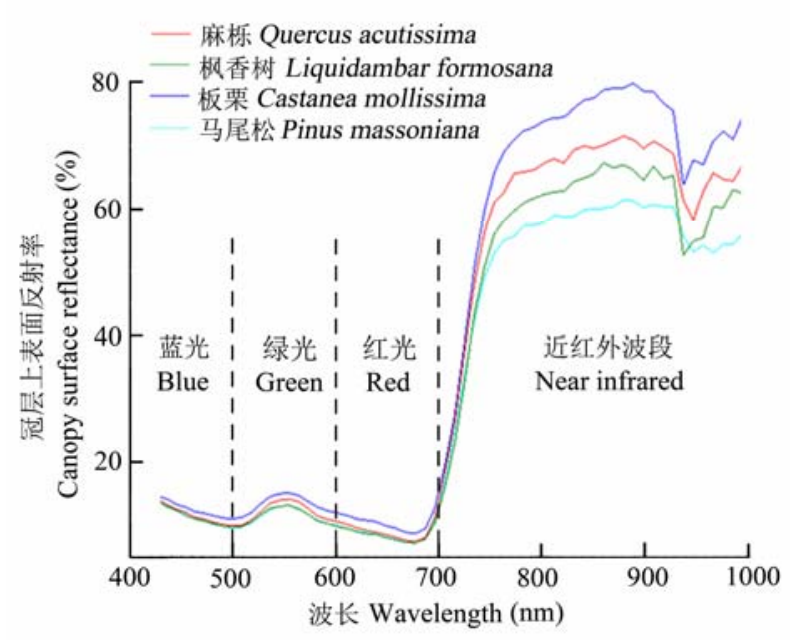

图2 4 个树种冠层的平均光谱反射率曲线。

Fig. 2 The mean reflectance value of tree clusters for four species.

行重分类, 并利用地面实测数据进行分类精度评 价。最后, 利用优化特征变量(12个)对森林类型再次 进行分类, 同样分为针叶和阔叶两种森林类型, 分 类方法使用BP神经网络法, 分类后加入单木冠幅信 息进行重分类, 并利用地面实测数据进行分类精度 评价。

\section{2 研究结果}

表2为单木冠幅提取的位置精度信息, 可见单 木冠幅提取位置准确性较高, 其总体精度达到 $81.4 \%$, 冠幅的探测率为 $77.3 \%$, 探测出的冠幅的准 确率为 $85.9 \%$ 。图3为提取的单木冠幅以及地面实测 冠幅半径与对应影像提取的冠幅半径生成的线性回 归方程; 拟合的直线接近于 $1: 1$ 线 $(k=0.901)$, 且截 距接近于 $0(b=0.002)$, 可见冠幅半径提取总体效果 较好。

表2 单木冠幅位置提取精度

Table 2 The accuracy of extracted crown position

\begin{tabular}{ccc}
\hline 探测率 & $\begin{array}{c}\text { 准确率 } \\
\text { Detection rate }\end{array}$ & $\begin{array}{c}\text { 总体精度 } \\
\text { Precision }\end{array}$ \\
\hline
\end{tabular}

百分比

77.3

85.9

81.4

总株数为 396 株。

The number of trees is 396 .

根据最大信息熵原理选取的信息熵前 6 个特征 变量分别为: MNF变换第一波段值(MNF1)、MNF变 换第三波段值(MNF3)、主成分变换第二波段值 (PCA2)、主成分变换第三波段值(PCA3)、土壤调节
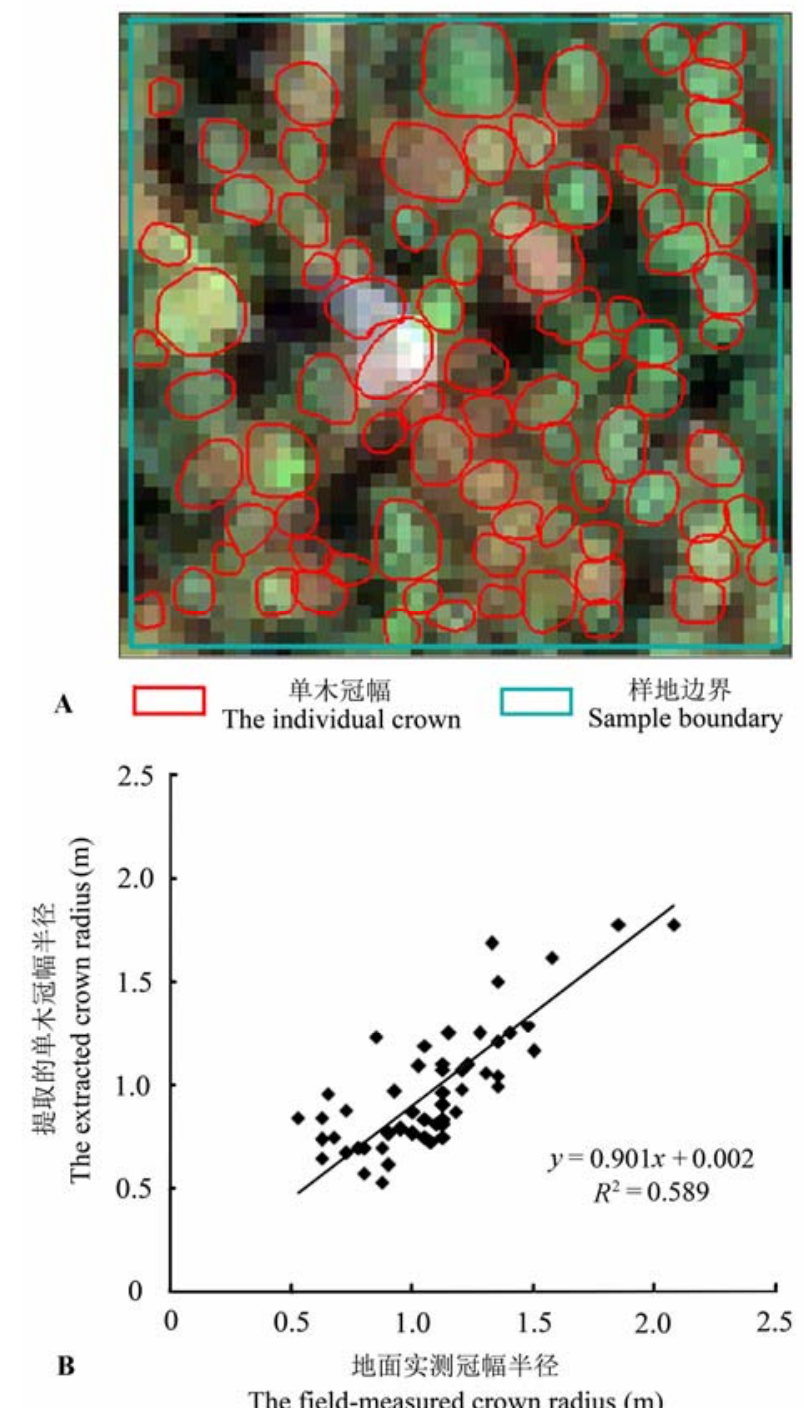

图3 面向对象分割方法提取的单木冠幅(A)及冠幅半径精 度验证(B)。

Fig. 3 The crowns extracted by object-oriented method (A) and accuracy assessment of extracted crown radius (B).

植被指数值(SAVI) 和波段组合特征VI $(40,15)$ (图4)。 由图4可见, MNF1可以较好地区分马尾松、麻栎(或 枫香树)和板栗; MNF3可以较好地将麻栎与其他 3 个数种区分开，同时也可以用于区分枫香树与马尾 松(或板栗); PCA2可较好地用于区分马尾松与其他 3 个树种; PCA3可以较好地将马尾松、麻柇和板栗 (或枫香树)区分开; SAVI可以将马尾松与麻栋完全 区分开, 另外也可以较好地用于区分麻栎与枫香 树; VI $(40,15)$ 可以较好地区分马尾松、麻柇和板栗 (或枫香树)。

表 3 为重分类后 4 个树种分类混淆矩阵。由表 3 可以看出四树种分类效果较好, 且利用全部特征变 

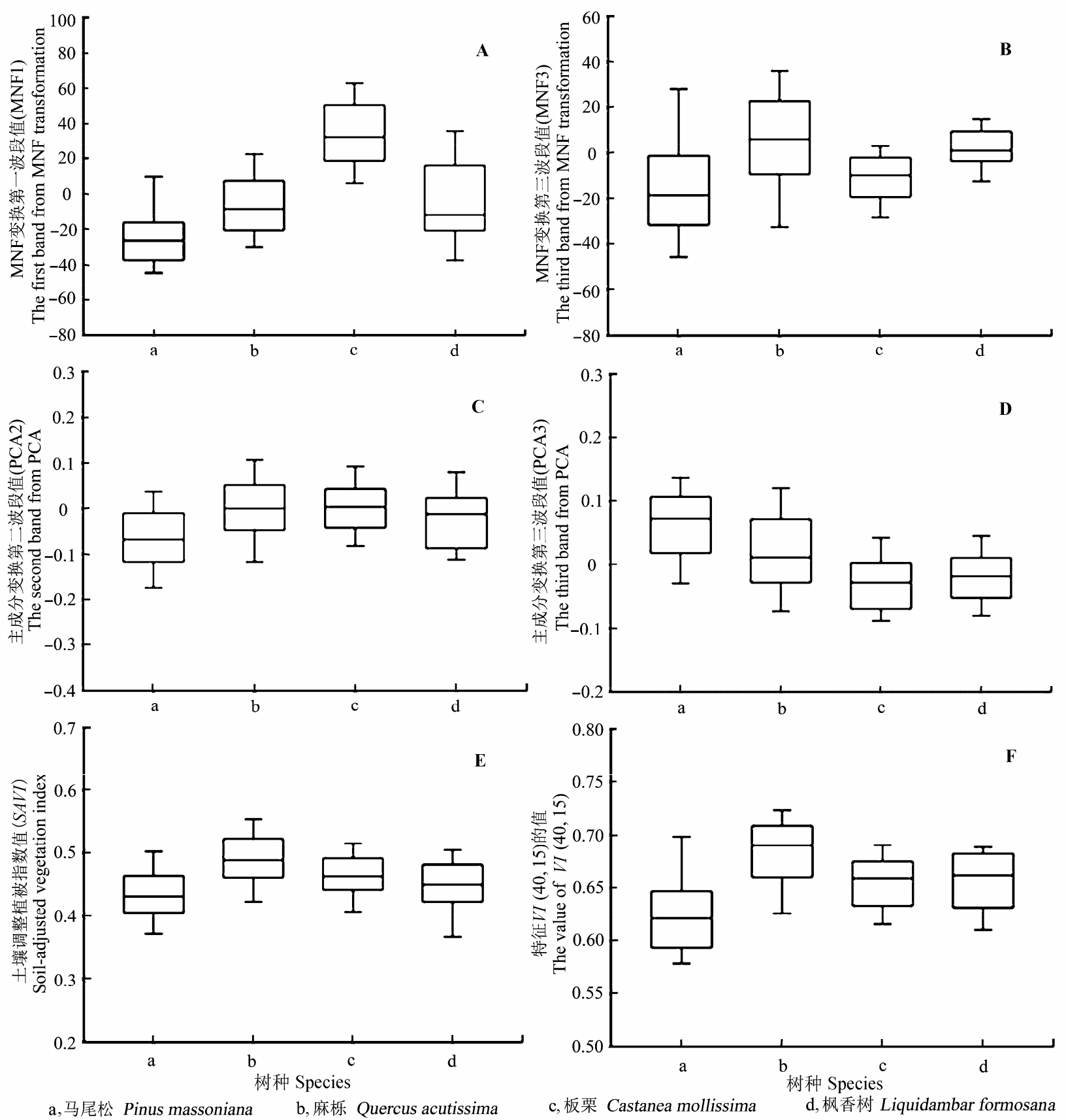

图4 重要特征变量图。A, MNF变换第一波段值(MNF1)。 B, MNF变换第三波段值(MNF3)。C, 主成分变换第二波段值(PCA2)。 $\mathbf{D}$, 主成分变换第三波段值(PCA3)。E，土壤调节植被指数值(SAVI)。F, 波段组合特征VI $(40,15)$ 。

Fig. 4 The diagram of important characteristic variables. A, The first band of MNF rotation (MNF1). B, The third band of MNF rotation (MNF3). C, The second band of principal components (PCA2). D, The third band of principal components (PCA3). E, Soil-adjusted vegetation index $(S A V I)$. F, The characteristic of band combination VI $(40,15)$.

量分类的结果略高于利用优化特征变量分类的结 果。利用全部特征变量 $(n=47)$ 分类时总体精度为 $64.6 \%$, Kappa系数为 0.493 , 其中板栗的分类精度最 高(75.0\%), 其次为麻栋(65.8\%), 最后为马尾松 (61.2\%)和枫香树(60.0\%); 利用选取的优化特征变 量 $(n=12)$ 分类时总体精度为 $62.9 \%$, Kappa系数为
0.459 , 其中板栗的分类精度最高(77.8\%), 其次为麻 栎(65.8\%), 最后为马尾松(58.6\%)和枫香树(51.0\%)。 表4为按森林类型分类结果的混淆矩阵(重分类 后)。由表4看出，按森林类型分类的精度高于 4 个树 种分类。利用全部特征变量 $(n=47)$ 分类时, 总体精 度为 $81.1 \%, \mathrm{Kappa}$ 系数为 0.584 , 其中阔叶树种分类 
表3 四树种分类混淆矩阵

Table 3 The confusion matrix of four species classification

马尾松 Pinus massoniana 麻栎 Quercus acutissima 枫香树 Liquidambar formosana 板栗 Castanea mollissima

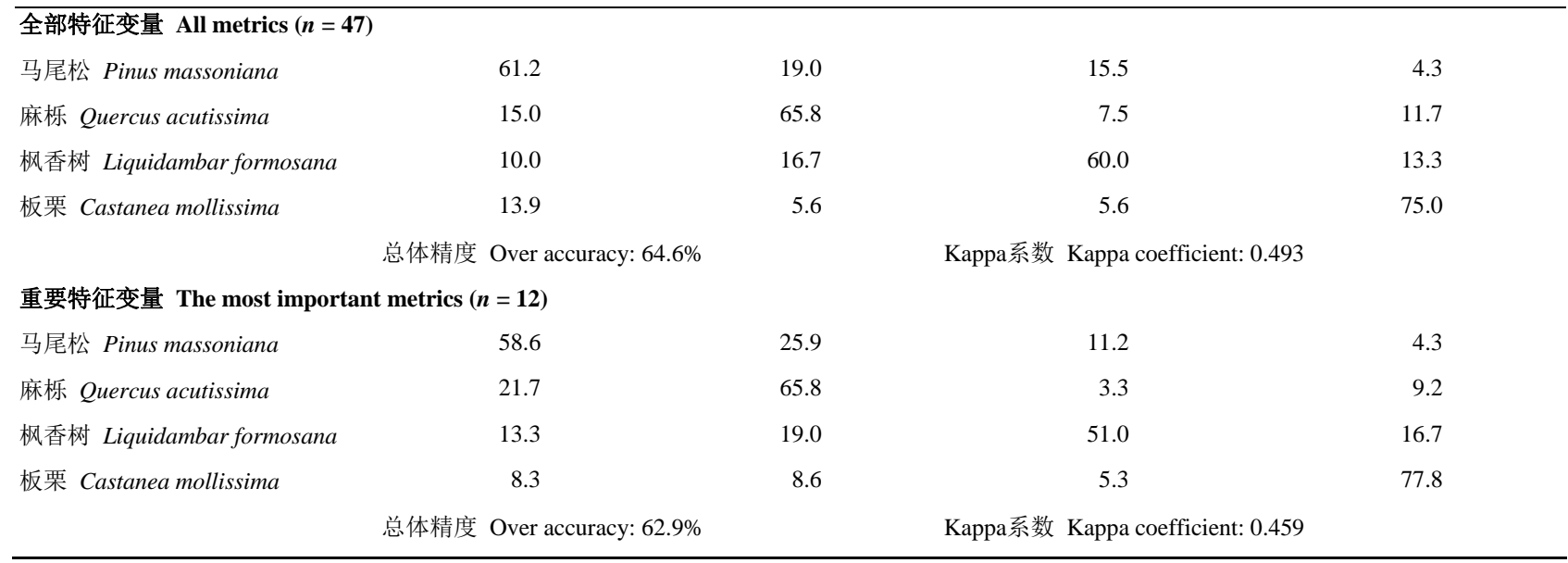

林木株数已转换为百分比。

The number of trees was converted to percentage.

表4 森林类型分类混淆矩阵

Table 4 The confusion matrix of the forest type classification

\begin{tabular}{cl}
\hline Coniferous trees & 阔叶树种 \\
\hline & Broadleaf trees \\
\hline
\end{tabular}

\begin{tabular}{|c|c|c|}
\hline \multicolumn{3}{|c|}{ 全部特征变量 All metrics $(n=47)$} \\
\hline 针叶树种 & 64.7 & 35.3 \\
\hline \multirow{3}{*}{$\begin{array}{l}\text { Coniferous trees } \\
\text { 阔叶树种 } \\
\text { Broadleaf trees }\end{array}$} & & \\
\hline & 8.6 & 91.4 \\
\hline & $\begin{array}{c}\text { 总体精度 } \\
\text { Over accuracy: } 81.1 \%\end{array}$ & $\begin{array}{c}\text { Kappa系数 } \\
\text { Kappa coefficient: } 0.584\end{array}$ \\
\hline \multicolumn{3}{|c|}{ 重要特征变量 The most important metrics $(n=12)$} \\
\hline 针叶树种 & 68.1 & 31.9 \\
\hline $\begin{array}{l}\text { Coniferous trees } \\
\text { 榈叶树种 }\end{array}$ & 16.2 & 83.8 \\
\hline Broaaleat trees & $\begin{array}{c}\text { 总体精度 } \\
\text { Over accuracy: } 77.7 \%\end{array}$ & $\begin{array}{c}\text { Kappa系数 } \\
\text { Kappa coefficient: } 0.525\end{array}$ \\
\hline
\end{tabular}

林木株数已转换为百分比。

The number of trees was converted to percentage.

精度为 $91.4 \%$, 针叶树种分类精度为 $64.7 \%$; 利用选 取的优化特征变量 $(n=12)$ 进行分类时, 总体精度为 $77.7 \%$, Kappa系数为 0.525 , 其中阔叶树种分类精度 为 $83.8 \%$, 针叶树种分类精度为 $68.1 \%$; 利用全部特 征变量分类的结果略高于利用优化特征变量分类的 结果，与 4 个树种分类的趋势相同。

研究以虞山林场为研究对象, 通过对集成传感 器 $\mathrm{LiCHy}$ 的同时相的高分辨率和高光谱影像以及外 业实测数据进行处理分析, 实现了北亚热带树种的 分类识别。研究表明, 利用全部特征变量进行 4 个典 型树种分类时, 总体精度为 $64.6 \%$, Kappa系数为 0.493; 而针对森林类型的分类精度为 $81.1 \%$, Kappa 系数为 0.584 ; 利用选取的优化特征变量分类精度
略低于利用全部特征变量的分类精度，其中对 4 个 典型树种分类时, 总体精度为 $62.9 \%$, Kappa系数为 0.459; 而针对森林类型的分类精度为 $77.7 \%$, Kappa 系数为 0.525 。

\section{3 讨论和结论}

本研究采用基于边缘检测的多尺度分割算法, 从多层次、多格局建立不同尺度的分割等级, 逐层 进行分割与信息提取, 相比传统的直接(在郁闭度 较低的林分影像上) 量测法(林辉等, 2004), 自动化 程度和精度都有所提升, 同时与复杂的空间统计学 理论(冯益明等, 2006)相比, 更易于推广。同时, 该 技术方案也有效地提供了冠幅位置和半径准确性的 验证精度, 为进一步的重分类提供了精度保证。

研究中通过最大信息熵原理选取的信息熵前 6 个特征变量, 即: MNF变换第一波段值(MNF1)、 MNF变换第三波段值(MNF3)、主成分变换第二波段 值(PCA2)、主成分变换第三波段值(PCA3)、土壤调 节植被指数值 $(S A V I)$ 和波段组合特征VI $(40,15)$ 。其 中, 最小噪声分离变换不仅可以使信息集中到少量 的低维数据中, 而且可以有效地分离出噪声信号 (Green et al., 1988), 因此通过最小噪声分离变换提 取的特征变量可以有效地代表原始影像的特征。杜 博等(2009)通过最小噪声分离变换提取特征变量进 行遥感影像分类取得了较好的结果, 与本文的研究 结果较为一致: 如图4所示, 在优化特征变量中, MNF1有效地将针叶树种和阔叶树种区分开, 对森 
林类型的分类有重要作用。而MNF2能够较好地区 分出马尾松和麻栎、板栗和枫香树, 对于树种的区 分也有重要意义。

主成分变换能够通过降维技术将多个指标转换 为少数几个彼此独立的综合指标，这些综合指标能 够反映原始指标的绝大部分信息。姚爱冬等(2014) 通过主成分变换提取影像特征因子反演地面参量取 得了较好的效果。研究也发现, 通过主成分变换提 取的PCA2和PCA3这两个特征变量较好地反映了树 种之间的差异(图4)。植被指数由于能够较为准确地 反映植被的理化特性, 经常被用作评价植被生长状 况、地表生物量和作物受灾害程度等的指标。在本 研究中针对各树种的特征波段区域建立了一系列植 被指数, 由图 4 可见, 植被指数在针叶和阔叶森林类 型以及阔叶树种之间有较好的区分。

研究采用 $\mathrm{BP}$ 神经网络算法对影像进行预分类, 并在预分类结果中加入单木冠幅信息进行影像的重 分类。结果表明: 在利用全部特征变量 $(n=47)$ 或优 化特征变量 $(n=12)$ 进行分类的情况下, 按森林类型 分类的精度都高于按 4 个树种分类的精度, 这是由 于森林类型特征变量的信息更为综合, 其差异性更 明显, 因而更准确; 按 4 个树种进行分类和按森林 类型进行分类时, 利用优化的重要特征变量的分类 结果都略低于全部特征变量的分类结果, 这表明特 征变量的篎选虽然会造成一定的信息损失, 但利用 优选的特征变量代替全部特征变量的方法是可行的 (即优选的特征变量可以很好地代表总体特征变量); 按森林类型分类时, 阔叶树种分类精度较高 $(n=47$ 时 $81.1 \% ; n=12$ 时 $77.7 \%$ ), 这是因为研究区的森林 类型处于次生演替阶段, 早期人工栽培的针叶树种 (如马尾松等)逐渐被本土阔叶树种(枫香树、麻栎等) 所代替, 阔叶树种生长旺盛, 占优势地位, 造成了 特征变量中阔叶树种的属性较丰富, 因而有利于阔 叶树种的识别。

本研究通过从高分辨率影像和高光谱影像中提 取丰富的冠幅特征和光谱信息, 对亚热带天然次生 林树种及森林类型进行分类, 取得了较好的分类精 度。研究使用的高分辨率传感器子系统采用超高空 间分辨率 $(0.1 \mathrm{~m})$, 有利于植被冠幅分割和边界识 别。高光谱传感器子系统的 64 个波段范围覆盖整个 可见光和近红外波段, 可以有效地捕获不同树种对 于特定波段(如红光: 620-740 nm, 近红外: 760-960 $\mathrm{nm}$ )波谱响应的细微变化; 另外其0.6 m的较高空间 分辨率和12 bit的较高量化级也增强了高光谱数据 的空间识别及辐射分辨能力。在实际应用中, 通过 该方法提取的森林树种信息可以对森林资源监测和 树种分布研究等提供定量化的空间数据支持, 也可 作为生态系统经营以及生态环境保护等的参考依 据。也可借助树种的精确提取和识别, 进行植被种 群格局分析, 发现群落形成和维持的潜在机制, 从 而深刻认识森林群落的生态学过程。同时, 精确的 树木空间分布和树种信息可为珍稀树种的保护以及 为森林生态系统的养分循环、碳流动和碳汇等研究 提供数据支撑。

亚热带次生混交林具有次生演替现象明显, 林 分垂直、水平结构复杂的特点(安树青和赵儒林, 1991), 植被冠层存在着垂直等级分层 (Popescu, 2007)。冠层按照所处的高度可分为优势层、共优势 层、中间层和覆被层, 在本研究中, 由于中间层和覆 被层的信息难以被传感器所探测, 因此只考虑优势 层和共优势层的冠幅, 这样在与地面实测数据进行 匹配验证时不可避免地带来了部分误差, 造成了冠 幅提取精度的降低。高分辨率和高光谱数据获得的 森林冠幅上表面二维(光谱)信息, 缺乏空间结构信 息, 且北亚热带天然次生林水平、垂直结构复杂, 次 生演替现象明显。在以后的研究中, 可尝试集成机 载激光雷达数据、高分辨率数据和高光谱数据, 以 期进一步提高北亚热带树种的分类精度。

基金项目 国家自然科学基金(31400492)、国家高 技术研究发展计划(863计划) (2013AA12A302)和 江苏高校优势学科建设工程资助项目。

致谢 感谢常熟市虞山国家森林公园的林业工作者 及南京林业大学林学院和园林学院部分研究生在野 外调查中提供的帮助。

\section{参考文献}

An SQ, Zhao RL (1991). Analysis of characteristics of secondary forest vegetation in the north subtropical zone of China. Journal of Nanjing University (Natural Sciences Edition), 27, 323-331. (in Chinese with English abstract) [安树青, 赵儒林 (1991). 中国北亚热带次生森林植被 的特征分析. 南京大学学报(自然科学版), 2, 323-331.]

Cao L, Coops NC, Hermosilla T, Innes J, Dai JS, She GH (2014). Using small-footprint discrete and full-waveform airborne LiDAR metrics to estimate total biomass and biomass components in subtropical forests. Remote 
Sensing, 6, 7110-7135.

Du B, Zhang LP, Li PX, Zhong YF (2009). A constrained energy minimization method in sub-pixel target detection based on minimization noise fraction. Journal of Image and Graphics, 14, 1850-1857. (in Chinese with English abstract) [杜博, 张良培, 李平湘, 钟燕飞 (2009). 基于 最小噪声分离的约束能量最小化亚像元目标探测方法. 中国图象图形学报, 14, 1850-1857.]

Feng YM, Li ZY, Zhang X (2006). Estimating forest stand crown based on high spatial resolution image. Scientia Silvae Sinicae, 42(5), 110-113. (in Chinese with English abstract) [冯益明, 李增元, 张旭 (2006). 基于高空间分 辩率影像的林分冠幅估计. 林业科学, 42(5), 110-113.]

Green AA, Berman M, Switzer P, Craig MD (1988). A transformation for ordering multispectral data in terms of image quality with implications for noise removal. IEEE Transactions on Geoscience and Remote Sensing Society, 26, 65-74.

Guo ZW, Li DM, Gan YL (2001). The assessment of forest ecosystem biodiversity by remote sensing. Acta Ecologica Sinica, 21, 1369-1384. (in Chinese with English abstract) [ 郭中伟, 李典谟, 甘雅玲 (2001). 森林生态系统生物 多样性的遥感评估. 生态学报, 21, 1369-1384.]

He MC (2006). Discussion on establishing a new mode of forest resources management with forest management plan as a platform and link. Forest Resources Management, (6), 4-11. (in Chinese with English abstract) [何美成 (2006). 以森林经营方案为平台和纽带建立森林资源管理新模 式的探讨. 林业资源管理, (6), 4-11.]

Li XM, Zhang QL, Li ZY, Tan BX (2010). The study on the forest types classification method of chris remote sensing image based on object. Journal of inner Mongolia Agricultural University, 31(2), 31-36. (in Chinese with English abstract) [李小梅, 张秋良, 李增元, 谭炳香 (2010). 基 于对象的CHRIS遥感图像森林类型分类方法研究. 内 蒙古农业大学学报, 31(2), 31-36.]

Li YH (2007). Experiment on using unmanned aerial vehicle in forest investigation. Forest Resources Management, (4), 69-73. (in Chinese with English abstract) [李宇吴 (2007). 无人机在林业调查中的应用实验. 林业资源管理, (4), 69-73.]

Lin H, Ning XB, Lü Y (2004). Compiling the standing volume table of Chinese fir based on the high-resolution satellite image. Scientia Silvae Sinicae, 40(4), 33-39. (in Chinese with English abstract) [林辉, 宁晓波, 吕勇 (2004). 基 于高分辨率卫星图像的立木材积表的编制. 林业科学,
40(4), 33-39.]

Luo JC, Zhou CH, Yang Y (2001). ANN remote sensing classification model and its integration approach with geo-knowledge. Journal of Remote Sensing, 5, 122-129. (in Chinese with English abstract) [骆剑承, 周成虎，杨艳 (2001). 人工神经网络遥感影像分类模型及其与知识集 成方法研究. 遥感学报, 5, 122-129.]

Pang Y, Li ZY, Ju HB, Liu QW, Si L, Li SM (2013). LiCHy: CAF's LiDAR, CCD and Hyperspectral airborne observation system. In: Silvilaser 2013, Proceedings of 2013 Silvilaser International Conference on Lidar Applications for Assessing Forest Ecosystems. Silvilaser, Beijing. 45-54.

Popescu SC (2007). Estimating biomass of individual pine trees using airborne lidar. Biomass and Bioenergy, 31, 646-655.

Wang J, Zhao TZ, Zeng Y (2013). Object-oriented classification of tree species based on rule extraction from rough set. Remote Sensing Information, 28(4), 90-97. (in Chinese with English abstract) [王婧, 赵天忠, 曾怡 (2013). 基于粗粘集规则提取的面向对象树种分类方法. 遥感 信息, 28(4), 90-97.]

Wang JB, Liu XS, Wu J (2013). Extraction technology of tree species information of hyperspectral remote sensing based on improved BPNN. Journal of Sichuan Agricultural University, 31, 264-268. (in Chinese with English abstract) [王吉斌, 刘晓双, 吴见 (2013). 基于改进BP神 经网络的高光谱遥感树种信息提取技术. 四川农业大 学学报, 31, 264-268.]

Wen YB, Fan WY (2013). Remote sensing image recognition for multi-temporal forest classification. Forest Engineering, 29(2), 14-20. (in Chinese with English abstract) [温一 博, 范文义 (2013). 多时相遥感数据森林类型识别技术 研究. 森林工程, 29(2): 14-20.]

Yao AD, Cao XY, Feng YM (2014). Remote-sensing model for estimating the size of gobi surface gravel based on principal components analysis. Journal of Desert Research, 34, 1215-1221. (in Chinese with English abstract) [姚爱冬, 曹晓阳, 冯益明 (2014). 基于主成分分析法的戈壁地表 砾石粒径遥感估测模型研究. 中国沙漠, 34, 1215-1221.]

Zhang DH, Lin Q (2000). The sustainable development of forestry and the Near-Nature forestry. Ecological Economy, (7), 23-26. (in Chinese) [张鼎华, 林卿 (2000). 近自然 林业与林业的可持续发展. 生态经济, (7), 23-26.]

责任编委: 朴世龙 责任编辑: 王 葳 
附录1 特征变量汇总表

Appendix 1 Table of characteristic variables

\begin{tabular}{|c|c|}
\hline 特征变量 Metrics & 变量描述 Description \\
\hline \multicolumn{2}{|l|}{ 原始单个波段 Initial bands } \\
\hline B38-39, B41-44, B48-53 & $\begin{array}{l}\text { 高光谱原始第38-39、41-44、48-53波段 } \\
\text { The 38-39, 41-44, 48-53 bands from hyperspectral data }\end{array}$ \\
\hline \multicolumn{2}{|l|}{ 波段组合 Band combination } \\
\hline$V I(39,52,53)$ & $\left(B_{39}+B_{52}+B_{53}\right) / 3$ \\
\hline$V I(42,38,53)$ & $\left(B_{42}+B_{38}+B_{53}\right) / 3$ \\
\hline$V I(43,38,53)$ & $\left(B_{43}+B_{38}+B_{53}\right) / 3$ \\
\hline$V I(44,38,53)$ & $\left(B_{44}+B_{38}+B_{53}\right) / 3$ \\
\hline$V I(51,38,39)$ & $\left(B_{51}+B_{38}+B_{39}\right) / 3$ \\
\hline$V I(41,38,31)$ & $\left(B_{41}-B_{38}\right) / B_{31}$ \\
\hline$V I(40,15)$ & $\left(B_{40}-B_{15}\right) /\left(B_{40}+B_{15}\right)$ \\
\hline$V I(45,31)$ & $B_{45}-B_{31}$ \\
\hline \multicolumn{2}{|l|}{ 植被指数 Vegetation index } \\
\hline 简单比值植被指数 Simple ratio vegetation index ( $S R$ ) & $B_{44} / B_{31}$ \\
\hline $\begin{array}{l}\text { 修正型简单比值植被指数 } \\
\text { Modified simple ratio vegetation index (MSR) }\end{array}$ & $\left(B_{39}-B_{6}\right) /\left(B_{34}-B_{6}\right)$ \\
\hline $\begin{array}{l}\text { 归一化植被指数 } 679 \\
\text { Normalized difference vegetation index } 679 \text { (NDVI-679 nm) }\end{array}$ & $\left(B_{44}-B_{31}\right) /\left(B_{44}+B_{31}\right)$ \\
\hline $\begin{array}{l}\text { 归一化植被指数 } 705 \\
\text { Normalized difference vegetation index } 705 \text { (NDVI-705 nm) }\end{array}$ & $\left(B_{39}-B_{34}\right) /\left(B_{39}+B_{34}\right)$ \\
\hline $\begin{array}{l}\text { 修正型归一化植被指数705 } \\
\text { Modified normalized difference vegetation index } 705 \text { (NDVI-705 nm) }\end{array}$ & $\left(B_{39}-B_{34}\right) /\left(B_{39}+B_{34}-2 B_{6}\right)$ \\
\hline $\begin{array}{l}\text { 土壤调整植被指数 } \\
\text { Soil-adjusted vegetation index (SAVI) }\end{array}$ & $\left(B_{44}-B_{31}\right) /\left(B_{44}+B_{31}+0.5\right)$ \\
\hline $\begin{array}{l}\text { 红边植被胁迫指数 } \\
\text { Red-edge vegetation stress index (RVSI) }\end{array}$ & $\left(B_{36}+B_{39}\right) / 2-B_{37}$ \\
\hline $\begin{array}{l}\text { 植被衰减指数 } \\
\text { Plant senescence reflectance index (PSRI) }\end{array}$ & $\left(B_{31}-B_{12}\right) / B_{39}$ \\
\hline $\begin{array}{l}\text { 植被水含量指数 } \\
\text { Water band index (WBI) }\end{array}$ & $B_{54} / B_{62}$ \\
\hline \multicolumn{2}{|l|}{ 数理统计特征 Statistical metrics } \\
\hline $\begin{array}{l}\text { 第一主成分 } \\
\text { First principal component }(P C 1)\end{array}$ & $\begin{array}{l}\text { 提取的主成分分析第一波段 } \\
\text { The first band from principal component analysis }\end{array}$ \\
\hline $\begin{array}{l}\text { 第二主成分 } \\
\text { Second principal component }(P C 2)\end{array}$ & $\begin{array}{l}\text { 提取的主成分分析第二波段 } \\
\text { The second band from principal component analysis }\end{array}$ \\
\hline $\begin{array}{l}\text { 第三主成分 } \\
\text { Third principal component (PC3) }\end{array}$ & $\begin{array}{l}\text { 提取的主成分分析第三波段 } \\
\text { The third band from principal component analysis }\end{array}$ \\
\hline $\begin{array}{l}\text { 独立成分分析第一波段 } \\
\text { First band of independent component analysis (ICA1) }\end{array}$ & $\begin{array}{l}\text { 提取的独立成分分析第一波段 } \\
\text { The first band from independent component analysis }\end{array}$ \\
\hline $\begin{array}{l}\text { 独立成分分析第二波段 } \\
\text { Second band of independent component analysis (ICA2) }\end{array}$ & $\begin{array}{l}\text { 提取的独立成分分析第二波段 } \\
\text { The second band from independent component analysis }\end{array}$ \\
\hline $\begin{array}{l}\text { 独立成分分析第三波段 } \\
\text { Third band of independent component analysis (ICA3) }\end{array}$ & $\begin{array}{l}\text { 提取的独立成分分析第三波段 } \\
\text { The third band from independent component analysis }\end{array}$ \\
\hline $\begin{array}{l}\text { 最小噪声分离变换第一波段 } \\
\text { First band of minimum noise fraction rotation (MNF1) }\end{array}$ & $\begin{array}{l}\text { 提取的MNF变换第一波段 } \\
\text { The first band from minimum noise fraction rotation }\end{array}$ \\
\hline $\begin{array}{l}\text { 最小噪声分离变换第二波段 } \\
\text { Second band of minimum noise fraction rotation (MNF2) }\end{array}$ & $\begin{array}{l}\text { 提取的MNF变换第二波段 } \\
\text { The second band from minimum noise fraction rotation }\end{array}$ \\
\hline $\begin{array}{l}\text { 最小噪声分离变换第三波段 } \\
\text { Third band of minimum noise fraction rotation (MNF3) }\end{array}$ & $\begin{array}{l}\text { 提取的MNF变换第三波段 } \\
\text { The third band from minimum noise fraction rotation }\end{array}$ \\
\hline \multicolumn{2}{|l|}{ 纹理特征 Texture metrics } \\
\hline $\begin{array}{l}\text { 相关度 } \\
\text { Correlation }(C R)\end{array}$ & $C R=\sum_{i, j=0}^{N-1} i \cdot P_{i, j}\left|\frac{(i-M E)(j-M E)}{\sqrt{V A_{i} \cdot V A_{j}}}\right|$ \\
\hline
\end{tabular}


附录1 (续) Appendix 1 (continued)

\begin{tabular}{|c|c|}
\hline 特征变量 Metrics & 变量描述 Description \\
\hline $\begin{array}{l}\text { 对比度 } \\
\text { Contrast (CO) }\end{array}$ & $C O=\sum_{i, j=0}^{N-1} n^{2} \cdot P_{i, j}$ \\
\hline $\begin{array}{l}\text { 相异性 } \\
\text { Dissimilarity (DI) }\end{array}$ & $D I=\sum_{i, j=0}^{N-1} i \cdot P_{i, j}|i-j|$ \\
\hline $\begin{array}{l}\text { 信息熵 } \\
\text { Entropy }(E N)\end{array}$ & $E N=\sum_{i, j=0}^{N-1} i \cdot P_{i, j}\left(-\ln P_{i, j}\right)$ \\
\hline $\begin{array}{l}\text { 均匀度 } \\
\text { Homogeneity }(H O)\end{array}$ & $H O=\sum_{i, j=0}^{N-1} i \cdot \frac{P_{i, j}}{1+(i-j)^{2}}$ \\
\hline $\begin{array}{l}\text { 平均值 } \\
\text { Mean }(M E)\end{array}$ & $M E=\sum_{i, j=0}^{N-1} i \cdot P_{i, j}$ \\
\hline $\begin{array}{l}\text { 二阶矩 } \\
\text { Second moment (SM) }\end{array}$ & $S M=\sum_{i, j=0}^{N-1} i \cdot P_{i, j}^{2}$ \\
\hline $\begin{array}{l}\text { 偏斜度 } \\
\text { Skewness (SK) }\end{array}$ & $S K=\frac{\left|\sum\left(P_{i, j}-M E\right)^{2}\right|}{\left(N^{2}-1\right)(V A)^{3 / 2}}$ \\
\hline $\begin{array}{l}\text { 方差 } \\
\text { Variance (VA) }\end{array}$ & $V A=\sum_{i, j=0}^{N-1} i \cdot P_{i, j}(i-M E)$ \\
\hline
\end{tabular}

